\title{
Spin-resolved off-specular neutron scattering from magnetic domain walls using the polarized ${ }^{3} \mathrm{He}$ gas spin filter
}

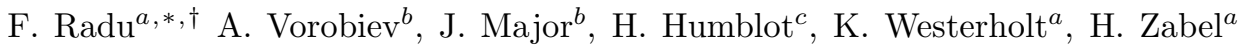 \\ ${ }^{a}$ Institut für Experimentalphysik/Festkörperphysik, \\ Ruhr-Universität Bochum, 44780 Bochum, Germany \\ ${ }^{b}$ Max-Planck-Institut für Metallforschung, Heisenbergstr. 3, D-70569 Stuttgart, Germany \\ ${ }^{c}$ Institut Laue-Langevin, 38042 Grenoble Cedex 9, France
}

\begin{abstract}
We report on the use of the polarized ${ }^{3}$ He gas filter and neutron resonant enhancement techniques for the measurement of spin-polarized diffuse neutron scattering due to ferromagnetic domains. A $\mathrm{CoO} / \mathrm{Co}$ exchange biased bilayer was grown on a $\mathrm{Ti} / \mathrm{Cu} / \mathrm{Al}_{2} \mathrm{O}_{3}$ neutron resonator template. The system is cooled in an applied magnetic field of $H_{a}=2000$ Oe through the Néel temperature of the antiferromagnet to $10 \mathrm{~K}$ where the applied magnetic field is swept as to measure the magnetic hysteresis loop. After the second magnetization reversal at the coercive field $H_{c 2}=+230$ Oe, the system is supposed to approach the original magnetic configuration. In order to prove that this is not the case for our exchange biased bilayer, we have measured four off-specular maps $\mathrm{I}++$, I +- , $\mathrm{I}-+, \mathrm{I}--$ at $H_{a} \approx+370 \mathrm{Oe}$, where the Co magnetic spins were mostly reversed. They show a striking behavior in the total reflection region: while the nonspin-flip scattering exhibits no diffuse reflectivity, the spin-flip scattering shows strong diffuse scattering at incident angles which satisfy the resonance conditions. Moreover the spin-flip off-specular part of the reflectivity is asymmetric. The I-+ intensity occurs at higher exit angles than the specularly reflected neutrons, and the I+intensity is shifted to lower angles. Their intensities are noticeably different and there is a splitting of the resonance positions for the up and down neutron spins $\left(\alpha_{n}^{+} \neq \alpha_{n}^{-}\right)$. Additionally, a strong influence of the stray fields from magnetic domains to the resonance splitting is observed.
\end{abstract}

PACS numbers: 75.60.Jk, 75.70.Cn, 61.12.Ha

Keywords: spin-resolved neutron reflectivity, off-specular scattering, ${ }^{3}$ He neutron spin filter, neutron resonator, exchange bias

The exchange bias (EB) phenomenon is associated with an exchange coupling between a ferromagnetic $(\mathrm{F})$ and an antiferromagnetic (AF) layer across their common interface, resulting in an unidirectional magnetic anisotropy and a shift of the magnetic hysteresis by an exchange bias field $H_{E B}$ [1]. A remarkable effect related to the exchange bias is the asymmetry of the magnetization reversal, which is best revealed by neutron scattering [2, 3, 4, 5. The first magnetization reversal after field cooling is dominated by domain wall movement, whereas all subsequent reversals proceed essentially by rotation of the magnetization. In the present work we report on neutron off-specular diffuse scattering experiments performed at applied magnetic fields close to the second coercive field $H_{c 2}$ supplementing the previous results at fields near the first coercive field $H_{c 1}$ [3].

The polarized ${ }^{3} \mathrm{He}$ gas spin filter was recently used for the first time [6] in a reflectivity experiment to measure diffuse scattering from thin magnetic films. The device itself is a cylindrical cell of $100 \mathrm{~mm}$ in length and $50 \mathrm{~mm}$ in diameter, filled with 0.5 bar ${ }^{3} \mathrm{He}$ gas. After filling,

\footnotetext{
*Permanent address: Department of Experimental Physics, National Institute of Physics and Nuclear Engineering, P. O. BOX MG-6, 76900, Magurele, Romania.

$\dagger$ Corresponding author. Tel: +49-234-322-4835; Fax: +49-234321-4173

Email address: Florin.Radu@rub.de (F. Radu)
}

the neutron polarization $P_{n}=P_{p} P_{3} H_{e}$ (where the $P_{n}$ is the measured neutron polarization, $P_{p}$ is the polarization of the polarizer and the $P_{3} \mathrm{He}$ is the polarization of the analyzer) was $65 \%$ and it decayed to about $59 \%$ in 24 hours. The transmission of the neutrons through the filter cell [7] is spin dependent with the following cross-sections: $\sigma_{\uparrow \uparrow}=5$ bn and $\sigma_{\uparrow \downarrow}=16500$ bn. In order to record the scattered intensities corresponding to all four neutron scattering cross-sections $(\mathrm{I}++, \mathrm{I}+-, \mathrm{I}-+$, I--), the experimental set-up includes, in addition, two Mezei spin flippers placed upstream and downstream of the sample [6]. The advantage of using the ${ }^{3} \mathrm{He}$ spin analyzer is that it is possible to register the spin-resolved diffuse scattering for a broad range of exit angles, and simultaneously it is free of additional spin-flip or small angle scattering, as it may occur for broad angle supermirror analyzers. However, there are drawbacks as well, namely that the cell absorption is not negligible and the flipping ratio seemed to be lower as compared to the case of the supermirror. In the present experiment these difficulties, especially the reduced neutron transmission, were successfully compensated by using an enhancement technique provided by the sample design as a neutron resonator [9, 10].

Recently (Nov-2002), the neutron polarization of the ${ }^{3} \mathrm{He}$ spin-filter reached an outstanding value of $94 \%$ [8] with a neutron transmission of about $40 \%$. Therefore the spin-flip ratio became comparable to the one provided by the supermirror. The experiment we report here was, 
however, performed on Sept-2001 when the ${ }^{3} \mathrm{He}$ did not have such high value of polarization.

The neutron resonances in thin films can be generated in two different ways: one way is in the total reflection region of a neutron resonator (bound states) and the other way is in a single film just above its critical angle for total reflection (quasi bound states), i. e. in the region of the Kissing fringes. In both cases the neutron density in the system is enhanced [9, 10] for definite incident neutron energies and one uses this phenomenon to increase the sensitivity to magnetic and nonmagnetic inhomogeneities and to non-collinear magnetic spin arrangements at the interface. As long as the observed effect takes place in the total reflection region, the scattered intensity is essentially the same as the incident intensity.

In the present experiments we used the neutron resonator to study the scattering from ferromagnetic domains in a $\mathrm{CoO}(25 \AA) / \mathrm{Co}(200 \AA)$ exchange biased bilayer. The bilayer was grown on a $\mathrm{Ti}(2000 \AA) / \mathrm{Cu}(1000 \AA) / \mathrm{Al}_{2} \mathrm{O}_{3}$ neutron resonator template. The enhancement of the neutron density takes place in the $\mathrm{Ti}\left(\rho_{\mathrm{Ti}}=-1.946 \cdot 10^{-6} \AA^{-2}\right)$ layer. The neutron scattering-length density $\rho$ is defined as $\rho=N b_{c o h}$, $N$ is the density of the scattering nuclei and $b_{c o h}$ is its coherent scattering length. The tail of the resonance enhanced neutron wave function extends over the $\mathrm{CoO} / \mathrm{Co}$ interface providing an increased sensitivity to its mag@push

The polarized neutron scattering scattering maps (Fig. 11) show a striking behavior in the total reflection region: while the nonspin-flip scattering shows weak diffuse reflectivity, the spin-flip scattering exhibits strong diffuse scattering at incident angles which satisfy the resonance conditions [9, 10]. Each resonance in such a system has different enhancement factors, decreasing from low orders $\left(\right.$ low $\left.\alpha_{i n}\right)$ to higher order (high $\alpha_{i n}$ ). One can easily observe in the I-+ map that the off-specular spikes are stronger in intensity for a large enhancement factor [9]. The I+- map shows a weaker intensity in the off-specular scattering signal, which is an effect of the different optical potential for $(+)$ and $(-)$ states, where the $(+)$ state $\left(\rho_{C o}^{+}=6.3 \cdot 10^{-6} \AA^{-2}\right)$ denotes the parallel orientation of the Co spins to the polarization direction and (-) state $\left(\rho_{\mathrm{Co}}^{-}=-1.78 \cdot 10^{-6} \AA^{-2}\right)$ denotes the antiparallel alignment of the Co spins in respect to the polarization direction. The enhancement factor for a (-) state is smaller than for $(+)$ state. This is caused by the transmission coefficient from vacuum through the top layers $(\mathrm{CoO}$ and $\mathrm{Co})$ to the resonant layer (Ti). For the $(-)$ states the transmission coefficient is higher than for the $(+)$ state and this affects strongly the enhancement factor for the $(+)$ and $(-)$ state resonances in the Ti layer.

The off-specular scattering is caused by the refraction on domain walls $[8]$. Assuming the domain walls to be perpendicular to the sample surface, the spikes will disappear and the off-specular intensity would be concentrated at their respective ends distinctly separated from the specular ridge $[8$. In order to explain a continu- netic properties. An important contribution to the increased sensitivity is the total-reflection region itself, provided by the $\mathrm{Cu}\left(\rho_{\mathrm{Cu}}=6.55 \cdot 10^{-6} \AA^{-2}\right)$ reflector layer.

The system is cooled in an in-plane applied magnetic field of $H_{a}^{F C}=+2000$ Oe through the Néel temperature of the ferromagnet to $10 \mathrm{~K}$, where the applied magnetic field $H_{a}$ is swept as to measure the magnetic hysteresis loop. After the second magnetization reversal $\left(H_{c 2}=+230 \mathrm{Oe}\right)$, the system is supposed to approach the original magnetic configuration. In order to prove that this is not the case for our exchange biased bilayer, we have measured four off-specular neutron reflectivity maps $\mathrm{I}++, \mathrm{I}+-, \mathrm{I}-+, \mathrm{I}--[$ Fig. 1] at $H_{a} \approx+370 \mathrm{Oe}$, where the magnetic spins were mostly reversed back to the direction of the applied field. In Ref. [3] the off-specular scattering (rocking curves at $\alpha_{\text {in }}+\alpha_{\text {out }}=$ constant) in the I+ - map was used to follow the development of the magnetic domains at the $\mathrm{CoO} / \mathrm{Co}$ interface before and after the first magnetization reversal to saturation and back to the remanence. In the present report we show measurements of the magnetic state of the EB system close but after the second magnetization reversal. The experiments were performed at the EVA reflectometer of the Institut Laue-Langevin (Grenoble, France) 11] with a neutron wavelength of $5.5 \AA$ using a $1 \mathrm{D}$ position sensitive detector (PSD).

ous spike we have to assume that the domains walls are continuously distributed. Furthermore, the orientation of the domain walls must spread from perpendicular to parallel to the sample surface. In a first approximation, the domain walls could be considered as the magnetic roughness at the $\mathrm{CoO} / \mathrm{Co}$ interface.

Another striking effect in Fig. 1 is the splitting of the position $\left(\alpha_{i n}\right)$ of the resonances by $0.68 \mathrm{mrad}\left(=.039^{\circ}\right)$. The magnitude of the splitting is too strong to be caused by the small applied field of $H_{a}=370$ Oe. The observed splitting requires an effective field of 1100 Oe [see Ref [12, 13]]. On the other hand, under the same experimental conditions of temperature and field, and before the first reversal takes place, i.e. no magnetic domains are created so far, we observe only a small splitting, which is consistent with an applied field of 370 Oe (not shown). Therefore, we need to assume that an additional stray field acts on the incoming neutrons and consequently on those which are inside of the spacer layer $(\mathrm{Ti})$. With this assumption the value of the splitting becomes feasible. The stray fields will not cause a spin-flip signal as they feature a slow gradient, but they will change the incoming and exit energies for the spin-flipped neutrons. Thus, we suggest that the stray fields from the magnetic domains are adding up to the applied field and the resulting Zeeman effect is the reason for the splitting we observed.

The additional field generated by the stray fields and the distribution of domain walls we observed leads us to the assumption that the interfacial magnetic domains 

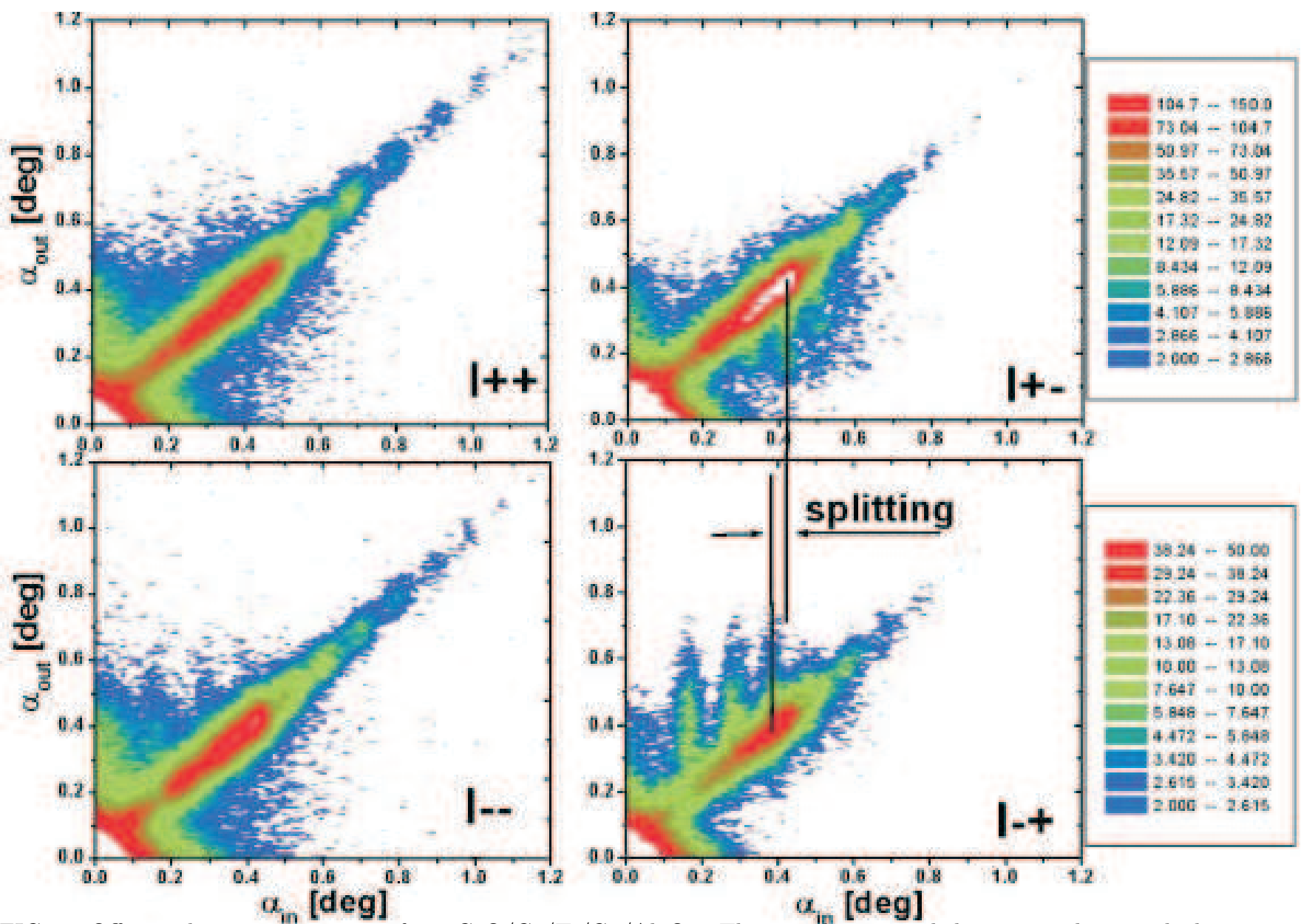

FIG. 1: Off-specular scattering maps from $\mathrm{CoO} / \mathrm{Co} / \mathrm{Ti} / \mathrm{Cu} / \mathrm{Al}_{2} \mathrm{O}_{3}$. The sample was cooled in an in-plane applied magnetic field of $H_{a}^{F C}=+2000$ Oe. At $\mathrm{T}=10 \mathrm{~K}$ the hysteresis loop was measured and the sample was set in a field of $H_{a}^{=}+370$ Oe, which is slightly higher than the second coercive field $\left(H_{c 2}=+230\right.$ Oe. The data it is not corrected for spin-flip efficiencies.

are ferromagnetic and that their magnetization vector is oriented perpendicular to the sample plane. The other possible case when the orientation of the magnetic domains were in-plane and perpendicular to the applied field would not cause the required stray fields for the splitting nor the type of domain walls necessary for generating the above described off-specular intensity distribution (spikes).

In conclusion we have used a polarized ${ }^{3} \mathrm{He}$ gas filter and neutron resonant enhancement techniques for the measurement of spin-polarized diffuse neutron scattering from ferromagnetic domain walls of an exchange biased $\mathrm{CoO} / \mathrm{Co}$ bilayer. After the first reversal domains are created at the $\mathrm{CoO} / \mathrm{Co}$ interface, which remain further on. We have shown that the magnetization of those domains points perpendicular to the interface and that their domain wall(s) is continuously distributed from perpendicular to parallel to the interface. We believe that the enhancement of off-specular scattering by using neutron resonators opens a new direction towards small angle neutron scattering (SANS) studies of magnetic and nonmagnetic thin films. The sensitivity to clusters, porous media, interface spin misalignment, magnetic domain walls, vortices, and inhomogeneities is under these conditions remarkably high.

This work is supported by the Deutsche Forschungsgemeinschaft (DFG), SFB 491.
[1] J. Nogués, I. K. Schuller, J. Mag. Mag. Mater. 192 (1999) 203.

[2] M. R. Fitzsimmons, P. Yashar, C. Leighton, Ivan K. Schuller, J. Nogues, C. F. Majkrzak and J. A. Dura, Phys. Rev. Lett. 84 (2000) 3986.

[3] F. Radu, M. Etzkorn, T. Schmitte, R. Siebrecht, A.
Schreyer, K. Westerholt, H. Zabel, J. Magn. Magn. Mater. 240 (2002) 251.

[4] M. Gierlings, M. J. Prandolini, H. Fritzsche, M. Gruyters, and D. Riegel, Phys. Rev. B 65 (2002) 092407.

[5] W.-T. Lee, S. G. E. te Velthuis, G. P. Felcher, F. Klose, T. Gredig, and E. D. Dahlberg, Phys. Rev. B 65 (2002) 
092407.

[6] B. Nickel, A. Rühm, W. Donner, J. Major, H. Dosch, A. Schreyer, H. Zabel, H. Humblot, Rev. Sci. Instr. 72 (2001) 163.

[7] F. Tasset, E. Ressouche, Nucl. Instr. and Meth. A 359 (1995) 537.

[8] F. Radu, A. Vorobiev, D. Jullien, H. Humblot, J. Major, F. Tasset, K. Westerholt, H. Zabel, to be published.

[9] F. Radu, V. K. Ignatovich, Physica B 292 (2000) 160.
[10] V. K. Ignatovich, F. Radu, Phys. Rev. B 64 (2001) 205408.

[11] H. Dosch, K. Al Usta, A. Lied, W. Drexel, and J. Peisl, Rev. Sci. Instrum. 63 (1992) 5533.

[12] G. P. Felcher, S. Adenwalla, V. O. De Haan. A. A. Van Well, Nature 377 (1995) 409.

[13] D. A. Korneev, V. I. Bodnarchuk, V. K. Ignatovich, J. Phys. Soc. Japan 65( Supplement A) (1996) 37. 